\section{Mit konsequenter Hygiene gegen multiresistente Keime}

— Der Patient Journey, also die Reise des Patienten durch das Krankenhaus, von der Aufnahme bis zur Entlassung, birgt ein hohes Infektionspotential - so infizieren sich jährlich etwa 500.000 Patienten ungewollt in deutschen Kliniken. Dabei wären mindestens 30\% dieser Infektionen durch verbesserte Hygienemaßnahmen vermeidbar, berichteten Experten anlässlich einer Veranstaltung des Bode Science Centers.

Besonders multiresistente Erreger (MRE) bergen ein hohes Mortalitätsrisiko - etwa 30.000 Patienten infizieren sich jährlich mit diesen Krankenhauskeimen. Dabei erweitert sich das Spektrum resistenter Problemkeime ständig - nicht nur Infektionen durch MRSA (Methicilinresistente Staphylococcus aureus), auch mehrfach resistente gramnegative Erreger (MRGN) bereiten Schwierigkeiten. Die Therapiemöglichkeiten schwinden hingegen, warnte Prof. Uwe Frank, Universitätsklinikum Heidelberg: „Wir kommen zunehmend in die Situation, dass wir schwere Infektionen durch multiresistente Erreger nicht mehr wirksam behandeln können. Handeln wir nicht jetzt, könnte die Sterblichkeit bei Infektionen bald wieder so hoch sein wie vor Einführung der Antibiotika". Im Fokus sollten ein gezielter Antibiotika-Einsatz sowie konsequent durchgeführte Hygienemaßnahmen stehen.

Postoperative Wundinfektionen: Sie sind die häufigsten nosokomialen Infektionen, gefolgt von Harnwegsinfektionen, unteren Atemwegsinfektionen und primären Sepsen. „Rund jede vierte nosokomiale Infektion ist eine postoperative Wundinfektion", erklärte PD. Dr. Andrej Trampuz, Charité Berlin. Erreger breiteten sich auch in künstlichen Gelenken aus und lagerten sich als Biofilm auf Prothesen ab. Bereits einfache Hygienemaßnahmen - wie Patienten vor einem Eingriff antiseptisch zu waschen - könnten helfen, die Infektrate deutlich zu senken.

Das Bode Science Center identifizierte wichtige Pflegemaßnahmen entlang des Patient Journey, bei denen Hygienefehler zum Tragen kommen und entwickelte dafür Standardarbeitsprozesse (SOPs). Ein besonderes Augenmerk lag auf häufigen und sicherheitsrelevanten Tätigkeiten: dem Legen peripherer venöser Zugänge und Harnwegskatheter, dem postoperativen Wechsel von Wundverbänden und dem Umgang mit Beatmungspatienten. Die Abläufe wurden in einzelne Arbeitsschritte gegliedert, auf Evidenz geprüft und in eine logische, gut nachvollziehbare Reihenfolge gebracht. Darüber hinaus ergänzen Checklisten, E-Learnings, Präsenzschulungen und E-Health-Applikationen das multimodale Konzept.

Eine Schlüsselposition nimmt die Händehygiene ein - nur jede zweite erforderliche Händedesinfektion werde auch durchgeführt, so Dr. Henning Mallwitz, Bode Science Center, Hamburg. Dies sei jedoch kein Wissens-, sondern ein Umsetzungsproblem. Studien konnten zeigen, dass bei Blutentnahme und Verbandwechsel die Einführung von SOPs zu einer Steigerung der Händehygiene von 65 auf $97 \%$ führte. Bei der Händedesinfektion vor aseptischen Tätigkeiten konnte die Compliance verdoppelt werden. Ein positives Fazit zog auch Claudia Becker, Fachkrankenschwester von der Pilotklinik St. Nikolaus-Stiftshospital GmbH, Andernach: Nur wenige Monate nach Einführung der SOP zum Wundverbandwechsel, stieg die Bereitschaft zur Einhaltung aller hygienerelevanten Schritte an. Zudem hätten sich die Infektionsraten grob halbiert und der Antibiotikaverbrauch sei um etwa $30 \%$ gesunken.

$(s p)$

Symposium „Antibiotikaresistente Erreger: Neue Hygienestrategien entlang der Patient Journey"; Berlin, 17. November 2016 (Veranstalter: Paul Hartmann AG/Bode Science Center)

\title{
Mistelextrakt steigert Lebensqualität
}

— Mistelextrakte gehören in Deutschland zu den meistverordneten Medikamenten bei Tumorpatienten, die die aktive Tumortherapie unterstützen.Es gibt gut belegte wissenschaftliche Evidenz, dass Mistelextrakte die Lebensqualität von Tumorpatienten, insbesondere von Patientinnen mit Mammakarzinom, während und nach einer konventionellen Tumortherapie verbessern und die Nebenwirkungen der antitumoralen Therapie vermindern können. Eine aktuelle Studie belegt die positiven Effekte von Mistelextrakt auf die Parameter Schlaf, Schmerz und Fatigue.
BREMISTAL ${ }^{\oplus} \mathrm{M} / \mathrm{P} / \mathrm{Qu}$ ist ein fermentierter wässriger Auszug aus Apfelbaummistel $(M)$, Kiefernmistel $(P)$ oder Eichenmistel (Qu), der subkutan appliziert wird. BREMISTAL ${ }^{\oplus} M$ wird vor allem bei prämenopausalen Patientinnen mit Mammakarzinom, BREMISTAL ${ }^{\oplus} \mathrm{P}$ bei postmenopausalen Patientinnen mit Mammakarzinom und BREMISTAL ${ }^{\oplus}$ Qu bei Patienten mit Prostatakarzinom empfohlen.

Der Mistelextrakt wird von den gesetzlichen Krankenkassen in der palliativen Tumortherapie erstattet und ist auch für die adjuvante Tumorbehandlung zugelassen. Hier hängt die Erstattung von den individuellen Satzungsleistungen der Krankenkasse ab.

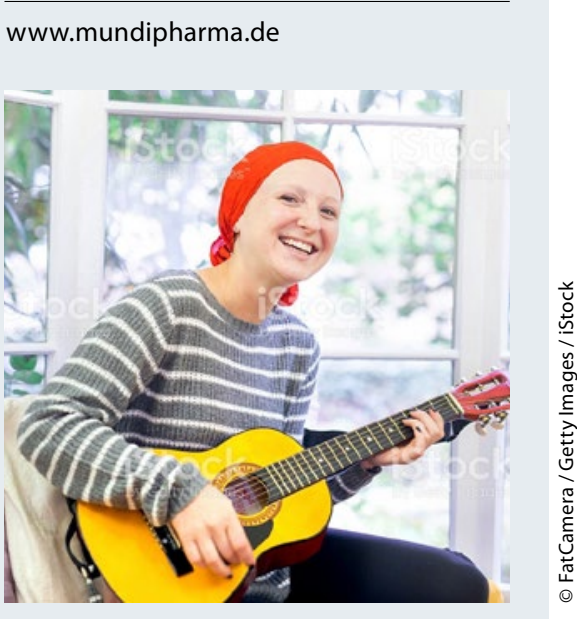

